\title{
APPLICATIONS OF MICROWAVES TO ANTIPROTON CONTROL
}

B. Autin, G. Carron, F. Caspers and L. Thorndahl

\begin{abstract}
A major achievement in particle accelerator physics has been the invention of stochastic cooling, a method which increases the density of beams of rare particles, like antiprotons, by several orders of magnitude. The beam circulates in a storage ring where it is sampled by electromagnetic devices which detect and correct the statistical fluctuations in position and energy. The efficiency is related to the sampling resolution which is itself associated with the system frequency bandwidth, a few gigahertz in practice. The coupling structures are made of electrode arrays connected by combiner or splitter networks. The dynamic range may exceed $150 \mathrm{~dB}$ yet fulfilling stringent linear characteristics. At the detection stage, the thermal noise is reduced using cryo-electronic techniques. At the other end of the amplification chain, solid state amplifiers delivering up to 100 watts $C w$ power have been preferred to travelling wave tubes for reasons of phase linearity, lifetime and economy. The performances and technological aspects of the microwave systems are discussed by the example of the CERN antiproton project, ACOL.
\end{abstract}

Invited paper, 17th European Microwave Conference Rome, Italy, 7th-10th September 1987 



\title{
APPLICATIONS OF MICROWAVES TO ANTIPROTON CONTROL
}

\author{
B. Autin, G. Carron, F. Caspers, L. Thorndahl*
}

\section{ABSTRACT}

A major achievement in particle accelerator physics has been the invention of stochastic cooling, a method which increases the density of beams of rare particles, like antiprotons, by several orders of magnitude. The beam circulates in a storage ring where it is sampled by electromagnetic devices which detect and correct the statistical fluctuations in position and energy. The efficiency is related to the sampling resolution which is itself associated with the system frequency bandwidth, a few gigahertz in practice. The coupling structures are made of electrode arrays connected by combiner or splitter networks. The dynamic range may exceed $150 \mathrm{~dB}$ yet fulfilling stringent linear characteristics. At the detection stage, the thermal noise is reduced using cryo-electronic techniques. At the other end of the amplification chain, solid state amplifiers delivering up to 100 watts $\mathrm{CW}$ power have been preferred to travelling wave tubes for reasons of phase linearity, lifetime and economy. The performances and technological aspects of the microwave systems are discussed by the example of the CERN antiproton project, ACOL.

\section{INTRODUCTION}

Ten years ago, the first beam of antiprotons, the heavy constituents of antimatter, circulated in a machine called ICE. Three years later, antiprotons were stored in a quasi-continuous way in the Antiproton Accumulator. The first antiproton factory was born 1 . These pioneering experiments took place at the European Laboratory of Particle Physics (CERN). They were followed by the Tevatron Project 2 at the Fermi National Accelerator Laboratory in Batavia, Illinois, USA and the LEAR project at CERN3. These facilities are dedicated to fundamental particle physics. Results of paramount importance have already been obtained with the discovery at CERN of the $W$ and $z$ particles which mediate the electroweak force, a synthesis of the electromagnetic and weak interactions which, for instance, explains the mechanism of the nuclear combustion in the sun or the explosion of the external layer of a supernova. Such discoveries, inevitably, trigger new questions which should be answered, at least partially, by the Tevatron and a new generation of experiments at CERN made possible with an upgraded antiproton source whose acronym, ACOL, stands for Antiproton Collector ${ }^{4}$.

The antiprotons are created during the impact of a high-energy proton beam on a solid target. The proton kinetic energy is converted into the creation of a few proton-antiproton pairs which emerge downstream of the target within a rather wide solid angle and with a large dispersion in energy. Such scarce particles produced in so diffuse a way are not amenable to efficient storage without special treatment dubbed "cooling" because it reduces the temperature of the antiprotons in the moving frame of their barycentre.

\footnotetext{
${ }^{\star}$ CERN, Geneva, Switzerland
} 
One type of cooling is based upon the Coulomb interaction between a well-collimated electron beam and the antiprotons, it is well suited for antiproton energies below a few hundreds of $\mathrm{MeV}$ and is even planned for the Soviet High-Energy Physics Complex, UNK. However, Coulomb coupling diminishes dramatically when the energy reaches the $\mathrm{GeV}$ range and the European and American antiproton sources rely on electromagnetic correction of the antiproton beam, the stochastic cooling technique.

\section{PRINCIPLES OF STOCHASTIC COOLING}

The principle of stochastic coolings consists of sampling a continuous, or, sometimes, bunched beam circulating in a storage ring and of reducing the deviation in position or momentum of each sample barycentre with respect to nominal values. The beam samples are detected by an array of electrodes, the pick-up and corrected by a similar device, the kicker (Fig. 1). The time delay of the electromagnetic signal is arranged to be equal to the propagation time of the particles from pick-up to kicker. If the samples were the same at each revolution, a stationary state would be obtained after one turn and the re-ordering of the beam would be modest. Fortunately, due to their different momenta, the particles do not all travel at the same velocity and the samples are partially randomized at each turn and pick-up traversal.

The description of stochastic cooling in the time domain is best transposed in the frequency domain not only because the formalism is better suited to certain theoretical aspects but also for the specification of the electromagnetic systems. In the frequency domain, a sample becomes a wave packet. The frequencies are distributed over a band whose width $w$ determines a characteristic cooling time $r$

$$
\tau=\frac{N}{W}
$$

for a beam containing $N$ particles:

The beam fluctuations have bands of frequency signals named the Schottky spectrum. A single particle rotating on its orbit at the frequency $f_{0}$ has a line spectrum of periodicity $f_{0}$ (Fig. 2):

$$
\mathrm{f}=\mathrm{nf}_{0}
$$

whereas a set of particles inside a finite momentum byte $\Delta p$ has a band spectrum for which each band has a width:

$$
\Delta f=n \frac{d f_{0}}{d p} \Delta p .
$$

In practice, the particles oscillate about their orbits, the signal is then modulated in amplitude and each harmonic has two side-bands. One notes that the bands are broadened at high frequency (Fig. 3); as it can be proven that cooling is optimum when the bands are separated, it results that an upper value of the frequency bandwith is determined by the nonoverlapping conditions of the schottky bands. The cooling manifests itself by shrinkage of the schottky bands (Fig. 4). 


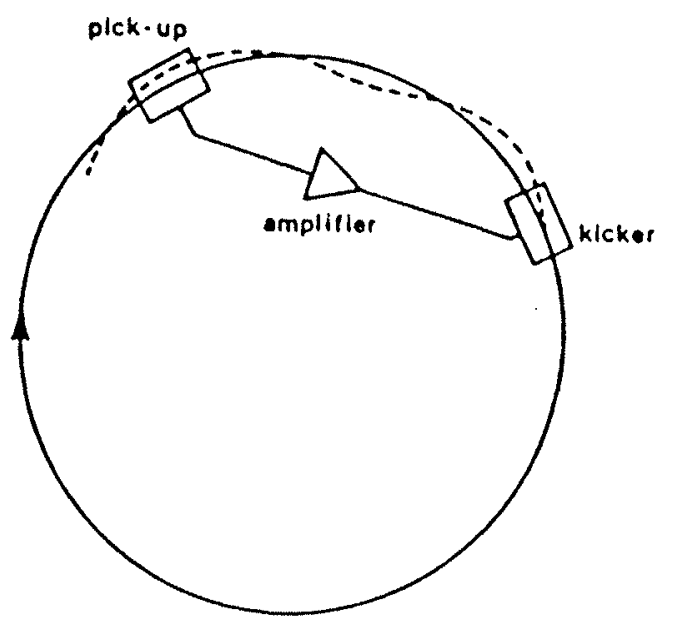

Eigure 1: Layout of a stochastic cooling system.

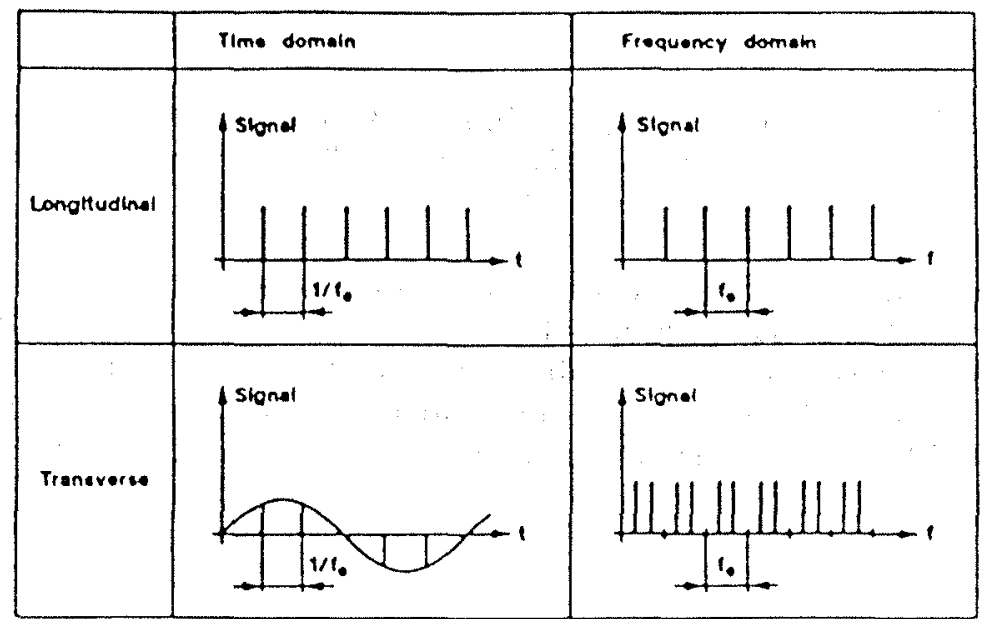

Eiqure 2: Schottky signals in time domain and frequency domain.

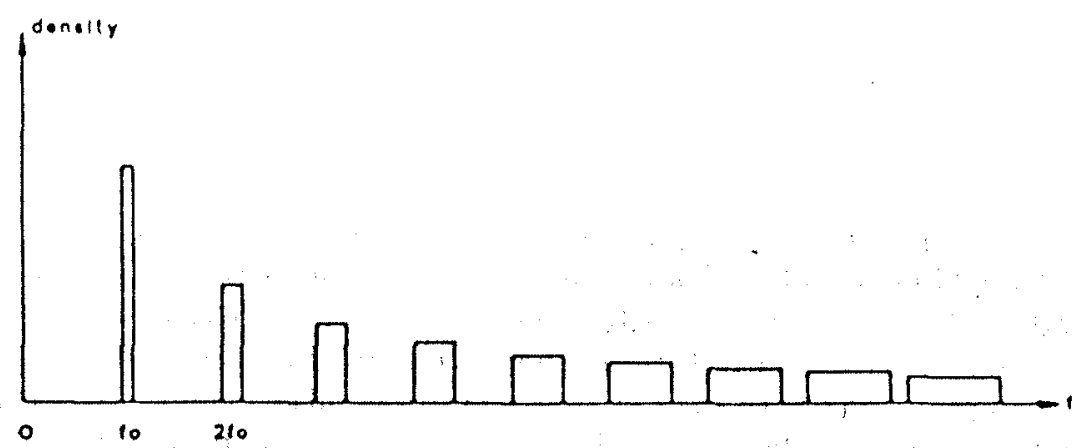

Eigure 3: Longitudinal Schottky bands originating from a large group of particles with slightly different revolution frequencies. At high frequencies the bands overlap. 


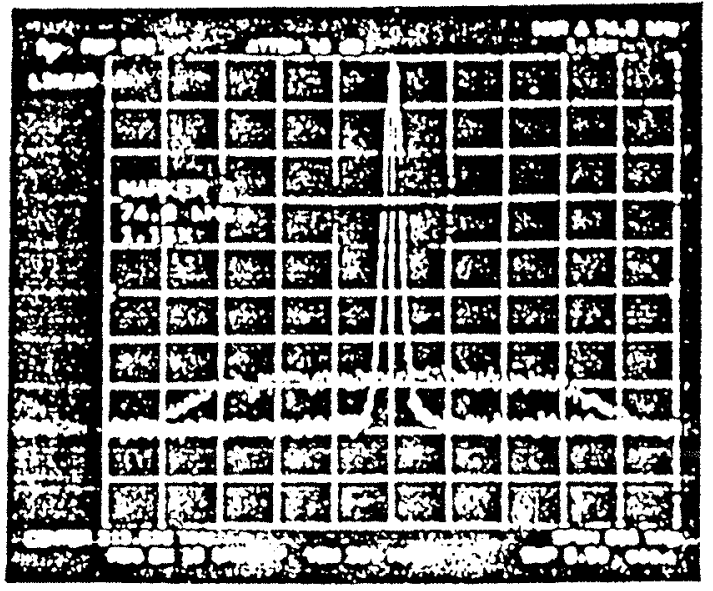

Fiqure 4: Beam spectral density before (flat distribution) and after (narrow distribution) momentum cooling.
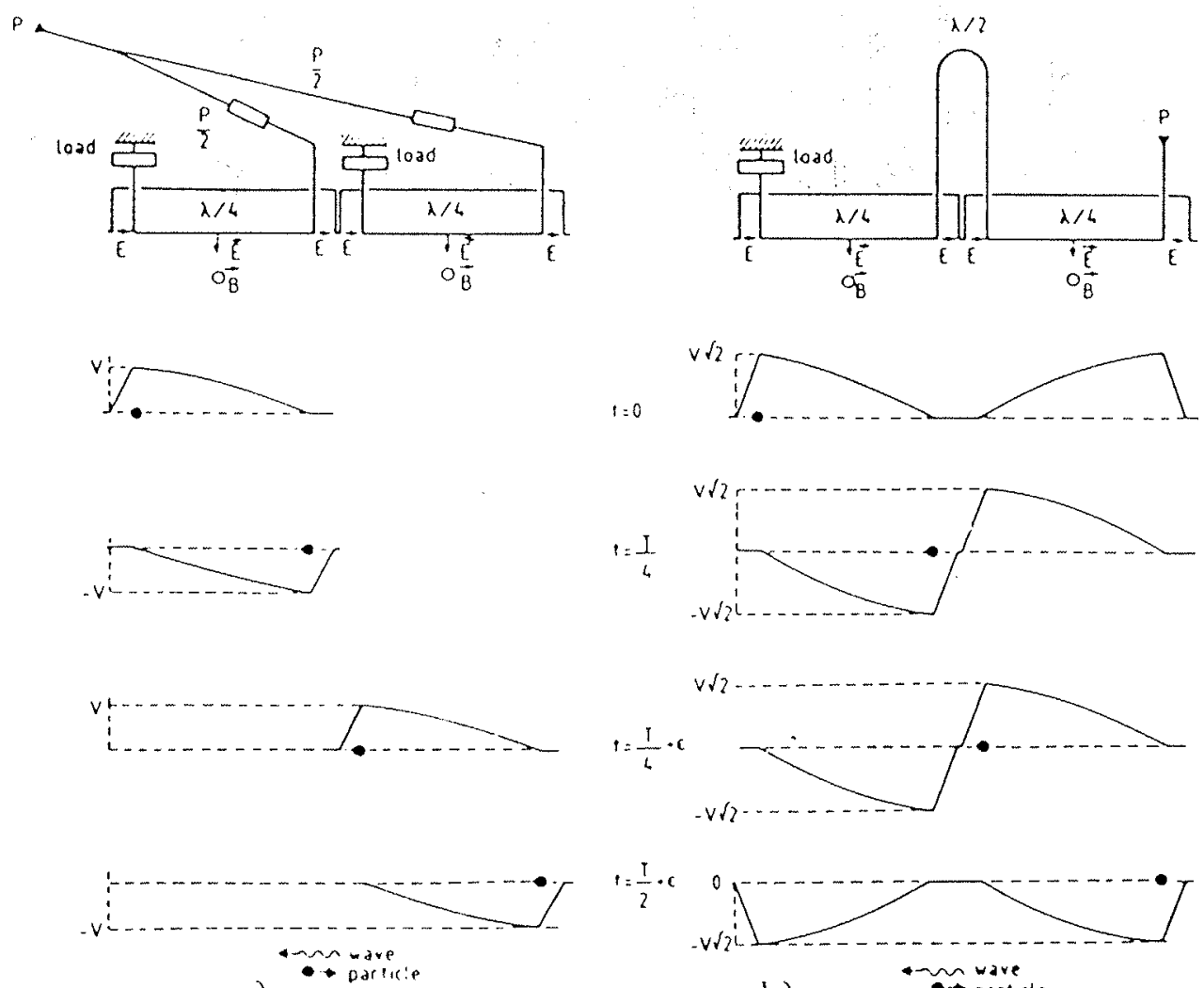

a)
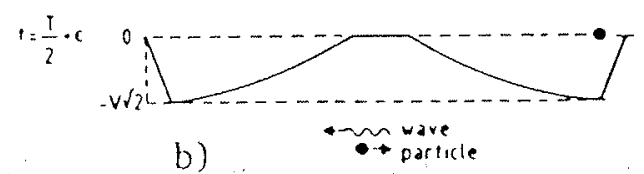

Fiqure 5: Wave-particle interaction along a strip-line electrode (a) or a super-electrode (b). 
The signal processing systems are characterized by their frequency response in amplitude (a) and phase $(\varphi)$. The most stringent condition concerns the group delay:

$$
t_{g}=\frac{d \varphi}{d \omega}
$$

which must be kept small with respect to the sample length. Smooth phase variations are compensated by phase equalizers but abrupt phase jumps have absolutely to be avoided.

In the ACOL project which is taken as an example, the ring circumference is $182 \mathrm{~m}$. The antiprotons of $3.5 \mathrm{GeV} / \mathrm{c}$ momentum have a velocity close to light velocity $(\beta=0.967)$ and their revolution frequency is $1.59 \mathrm{MHz}$. Their momentum spread is $\pm .75 \%$ and the momentum dependence of the revolution frequency dfo/dp is $8.3 \times 10^{6} \mathrm{~Hz} / \mathrm{eV}$. The frequency range extends from 1 to $3 \mathrm{GHz}$ and is split into three equal sub-bands. The sample length is smaller than 250 ps and contains 40,000 antiprotons at the most. The maximum width of a schottky band is $0.8 \mathrm{MHz}$. The sample population is fully renewed after 15,000 turns or $9 \mathrm{~ms}$. The characteristic cooling time, $50 \mathrm{~ms}$, would be obtained if the gain of the amplification chain was optimum; in fact, such a gain implies an exceedingly high output power and the system is operated at a much lower gain compatible with a cooling time constant of $1 \mathrm{~s}$.

\section{COUPLING BETWEEN BEAM AND ELECTRODES}

There exist a variety of methods to couple the electromagnetic field generated by charged particles with detection devices or, conversely, to transmit external electromagnetic power to a particle beam. As a general rule, the reciprocity principle is applied: any structure used as a pick-up can also act as a kicker and vice versa. A review of the methods used to couple particle beams with electromagnetic devices is given in Ref. 6 . Here, the description is limited to the stripline electrode for which the mechanism of the interaction is shown in Fig. 5 in the case of a kicker. For the longitudinal motion, a particle which grazes the electrode is accelerated, or decelerated, in the gaps where the electric field is parallel to the direction of motion. In the case of the transverse motion, the interaction takes place all along the electrode where the fields are perpendicular to the direction of motion. The electromagnetic wave (TEM mode) travels against the particle so that the deflections applied to the particles are always additive. In the case of a pick-up, the signal travels also against the particle and is therefore detected at the upstream end of the electrode whereas the downstream end is terminated by a load whose impedance is the characteristic impedance of the line $z_{L}$.

A longitudinal kicker can be characterized by a coefficient

$$
K_{n}=\frac{\Delta E}{e V_{K}}
$$

which measures the ratio of energy $\Delta \mathrm{E}$ gained by the particles to the input voltage $v_{K}$ delivered by the amplifier. In fact, the electrodes are placed symmetrically with respect to the beam and the voltage which is actually seen by the particles is 


$$
v_{\mathrm{L}}=\sqrt{\frac{\mathrm{Z}_{\mathrm{L}}}{2 \mathrm{R}_{0}}} \mathrm{v}_{\mathrm{K}}
$$

where $R_{0}$ is the amplifier output impedance $(50 \Omega)$. When $n$ pairs of electrodes are in parallel on the same amplifier, the voltage is divided by $\sqrt{n}$ and the coefficient $K_{n}$ is

$$
K_{*}=\sqrt{\frac{n Z_{L}}{2 R_{0}}} 2 g_{n} e^{j(\pi / 2-\theta)} \sin \theta .
$$

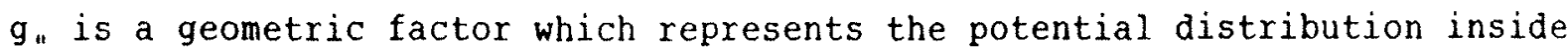
a pair of opposite electrodes, it is calculated by the methods of conformal mapping used for static fields

$$
g_{n}=\frac{2}{\pi} \tan ^{-1}\left(\sinh \frac{\pi w}{2 h}\right)
$$

where $w$ is the electrode width and $h$ the gap height, $\theta$ is a phase which relates the particle and wave velocities $v$ and $v_{L}$ with the frequency $\omega / 2 \pi$ for an electrode of length $\ell$

$$
\theta=\frac{1}{2}\left(\frac{1}{v}+\frac{1}{v_{L}}\right) w \ell
$$

The efficiency is maximum when $\theta$ is equal to $\pi / 2$. These electrodes are currently used for an octave bandwidth, their length is tuned to a quarter of the mid-frequency wavelength so that $\theta$ varies from $\pi / 3$ to $2 \pi / 3$. One notes that the phase of the kicker response is

$$
\varphi=\frac{\pi}{2}-\theta
$$

and that the group delay is constant.

In the method which has just been described, it is the electromagnetic power transferred to the beam which is added at each passage through an electrode gap. The efficiency would be increased if the voltage, instead of the power, was summed over the length of the electrode array. This can be done by connecting two consecutive loops with a half-wavelength line and one can show that the new coefficient $k_{\text {" }}$ becomes

$$
K_{n}=\sqrt{\frac{Z_{L}}{2 R_{0}}} 2 g_{n} e^{j n(\pi / 2-\theta)} \operatorname{tg} \theta \sin n\left(\frac{\pi}{2}-\theta\right) \cdot
$$

At the mid frequency where $\theta$ is still $\pi / 2$, the efficiency is increased by $\sqrt{n}$ with respect to the single electrode case but the improvement has to be paid by a bandwith reduction. This solution has been retained for the ACOL project where electrodes are connected in groups of two. 
A longitudinal pick-up is characterined by its transfer impedance

$$
z_{p}=\frac{v_{B}}{I_{B}}
$$

where $I_{B}$ is the particle current and $v_{B}$ the voltage delivered at the output of the electrode array on a nominal impedance $R_{0} ; V_{B}$ is conjugate to $V_{K}$ in the pick-up kicker correspondance. As a consequence of Lorentz reciprocity theorem, it turns out that

$$
z_{p}=\frac{1}{2} R_{0} K_{\mu}
$$

In contrast with the longitudinal case, the opposite electrodes for transverse kickers have opposite polarities so that the fields are zero on the centre line and vary linearly with the transverse position. The efficiency is then given by

$$
K_{\perp}=\frac{\Delta p_{\perp} v}{e v_{K}}
$$

where $\Delta \mathrm{p}$ is the change of transverse momentum. Its expression

$$
K_{\perp}=\sqrt{\frac{n Z_{L}}{2 R_{0}}} 4 g_{\perp} \frac{\ell}{h}\left(1+\frac{v}{v_{L}}\right) e^{-j \theta} \frac{\sin \theta}{\theta}
$$

for $n$ pairs of single electrode is derived in [6] and its generalization to the case of a superelectrode made of $n$ single electrodes gives

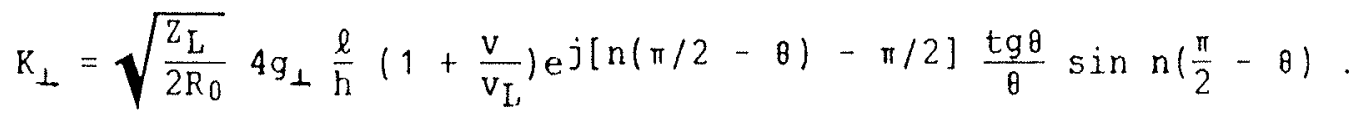

The change in polarity gives a new potential configuration

$$
g_{\perp}=\tanh \frac{\pi w}{2 h}
$$

Due to the presence of $\theta$ at the denominator, the efficiency is shifted towards low frequencies. The transfer impedance of a transverse pick-up is

$$
z_{p_{\perp}}=-\frac{1}{2} j \frac{\omega}{v} R_{0} K_{\perp}
$$

There is no more $\theta$ dependence at the denominator and the efficiency peaks at mid-frequency. Both for kicker and pick-up, the efficiency increases when the gap height $h$ is small. When a stochastic system is power limited, it is therefore advantageous to maintain the electrodes near the beam envelope during the cooling. As a counterpart, the line impedance $z_{L}$ diminishes when opposite electrodes are too close to each other. Nevertheless, in spite of the mechanical complication of having mobile structures under vacuum, the method is attractive because it makes an optimum use of the electronic power 7 and it has been adopted in the ACOL project (Fig. 6). 


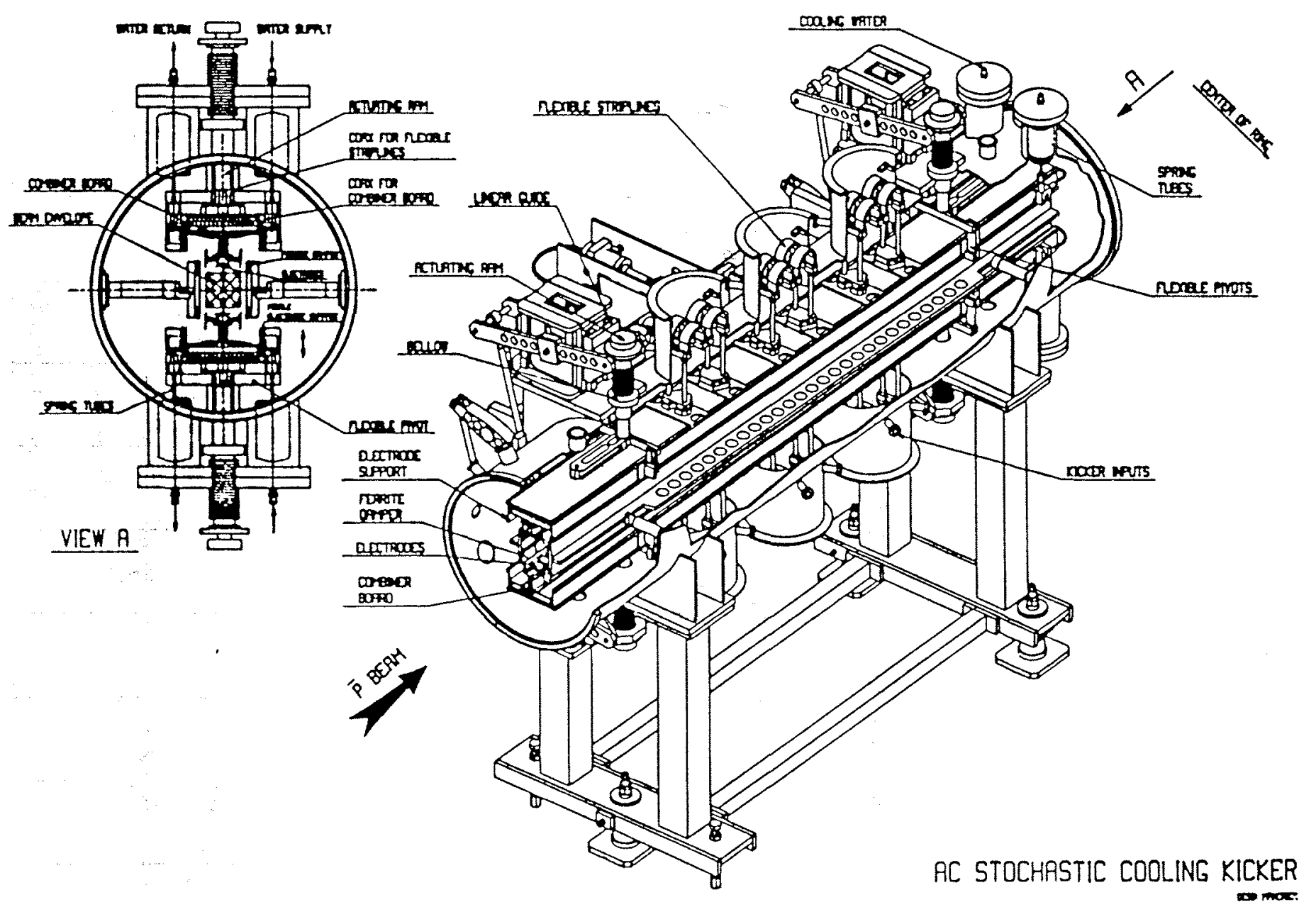

Eiqure 6: Cut-away diagram of ACOL stochastic cooling kicker. 


\section{SIGNAL PROCESSING}

A stochastic cooling system, especially when its cooling time is short (about $1 \mathrm{~s}$ ), has to cope with two extreme situations: to detect exceedingly weak beam fluctuations on one hand and to deflect high energy particles on the other hand. The signals have therefore to be integrated with all possible ingenuity. As it has been already mentioned, pick-up and kicker have reciprocal functions and their structure is very similar; for this reason, signal pocessing will be mainly described for the pick-up.

The number of electrodes or superelectrodes in a pick-up is determined by the signal to noise power ratio which is optimum near unity. When the pick-up length is not compatible with the space available in the storage ring, the background noise is reduced by thermal cooling of the structures. In ACOL, a thermal shield surrounds the pick-up and absorbs the radiation until an equilibrium temperature close to $1000 \mathrm{~K}$ is reached. In addition, the loads and the preamplifiers are refrigerated at $400 \mathrm{~K}$ in a special cold pot. The signals delivered by the various electrodes are usually combined outside the vacuum tank but, in a cold environment, the thermal leaks have to be minimized and a great part of the signal processing takes place in vacuo. The electrodes downstream ends are connected to a common load and the upstream ends to the same low-noise preamplifier through identical circuits which have a tree structure. Each branch has two functions: electrical delay and impedance matching using one or several $1 / 4$ wavelength transformers depending on the frequency bandwidth. In a pick-up tank, three levels (Fig. 8) can be considered, a combiner board near the electrodes, coaxial lines which transport the signals to a final impedance transformer which has the shape of a modified exponential stripline.

The combiner board is made of silver microstrips deposited on an alumina substrate. The topology of the strips, their shape and the masks were obtained using MICROS4 CAD program7. The difference electromagnetic modes due to unavoidable unbalances between adjacent lines are damped in wilkinson resistors. In order to meet the electrical and vacuum compatibility requirements, the thick layer technology turned out to be best suited. After layer deposition by serigraphy, the boards were fired at $850^{\circ} \mathrm{C}$ in a two-day temperature cycle. This long process is imposed by the dimensions of the alumina plates: $170 \times 400 \times 3 \mathrm{~mm}$. Due to their large dimensions and by analogy with the techniques currently used in microelectronics, such combiners can be called macrocircuits.

The tank size exceeds the electromagnetic wavelength by far and spurious cavity modes are excited. These modes spoil the quality of the system response and are therefore damped by resistive layers on the board itself $(R=108)$ and in ferrite tiles clamped on metallic walls located on each side of the particle beam. The ferrite is a Nickel-Zinc composite material which outgases little and keeps its magnetic properties at low temperature.

The kicker tank (Fig. 7) resembles the pick-up tank but works at room temperature. The electrode supports are water cooled to eliminate the heat of the loads into which the electromagnetic power is dumped.

Needless to say, the adjustment of such systems requires numerous laboratory experiments and a careful testing at all the stages of the 


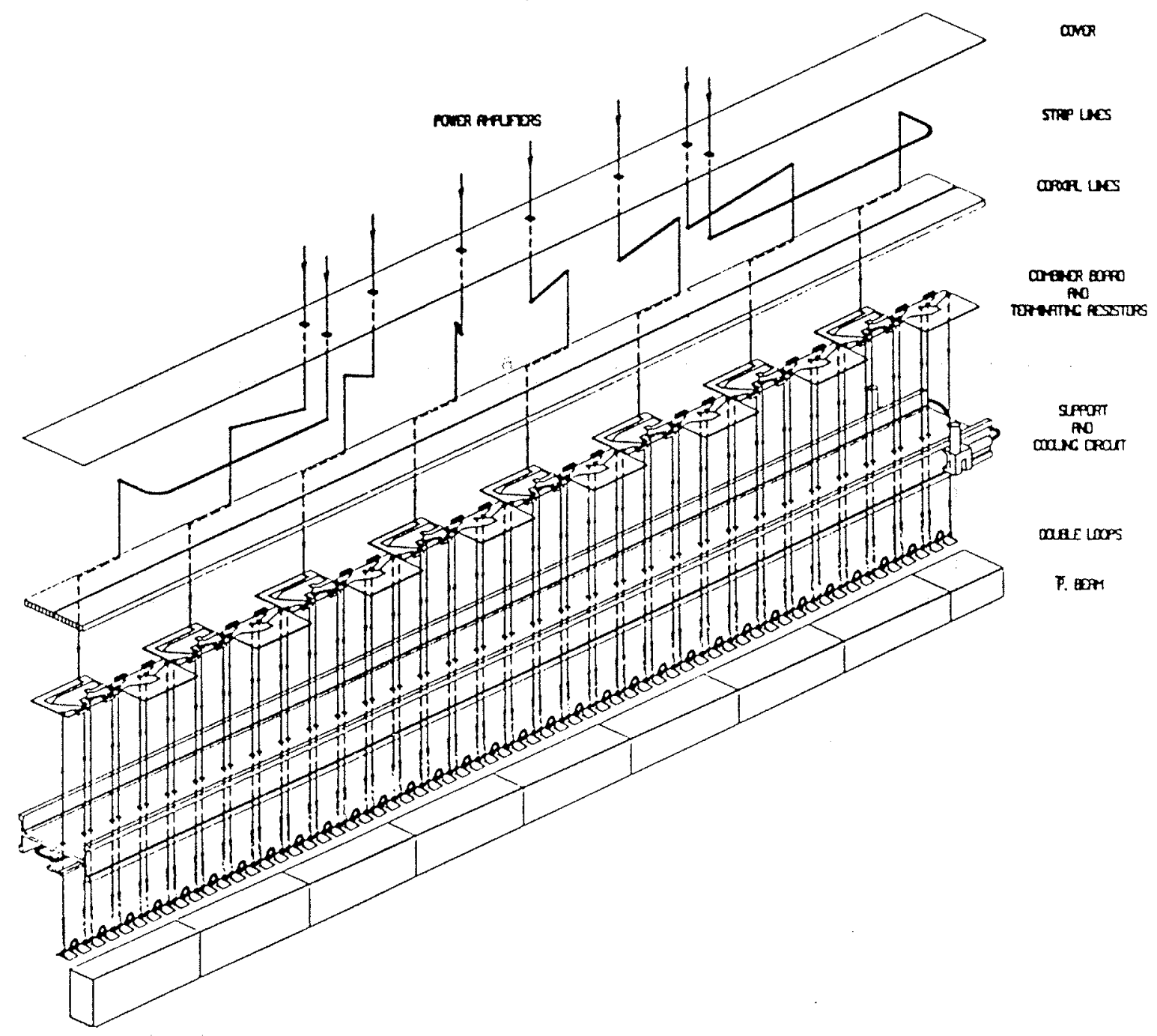

Fiqure 7: Blown-up view of the signal processing system in an ACOL kicker. 


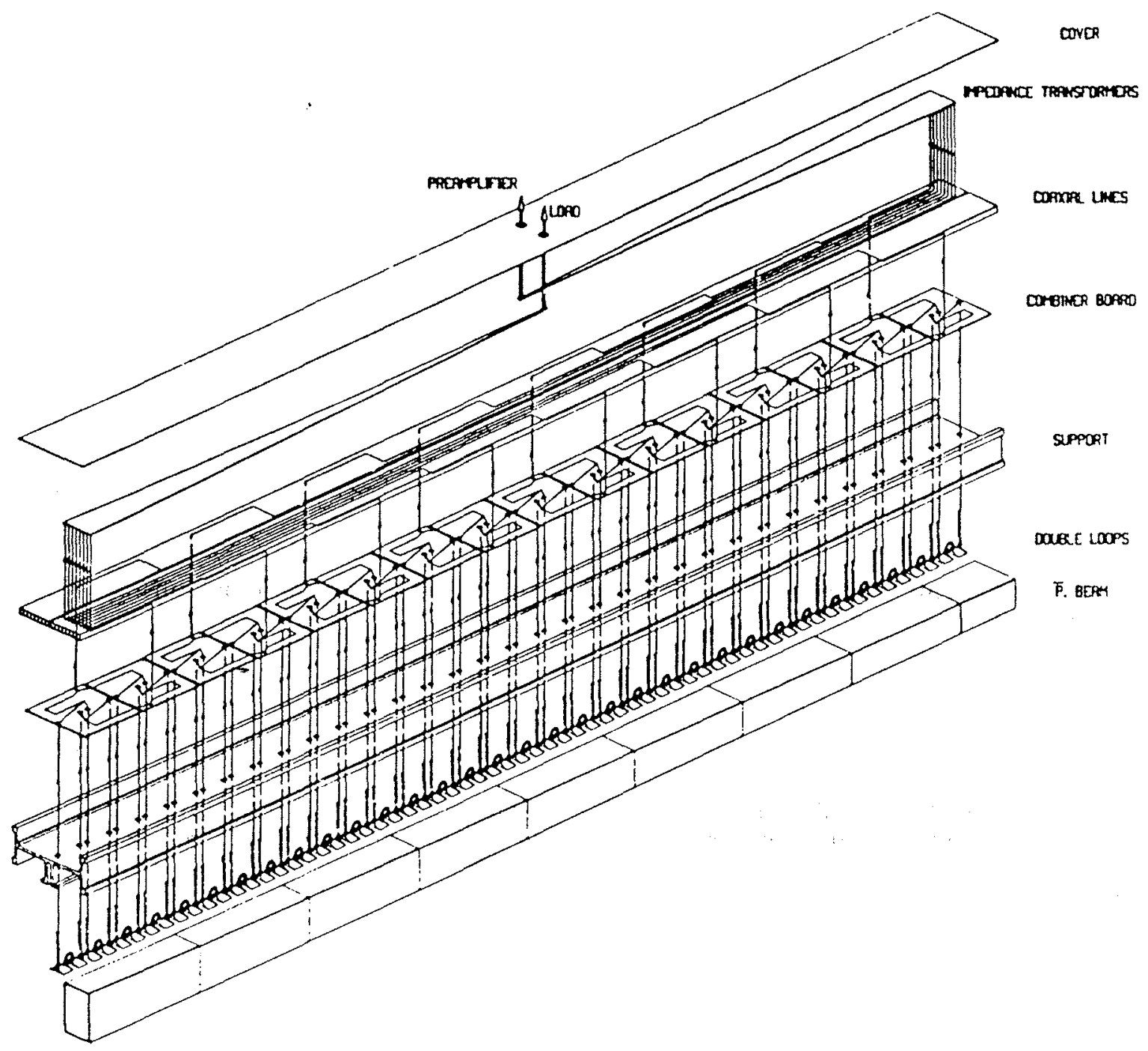

Eiqure 8: Blown-up view of the signal processing system in an ACOL pick-up.

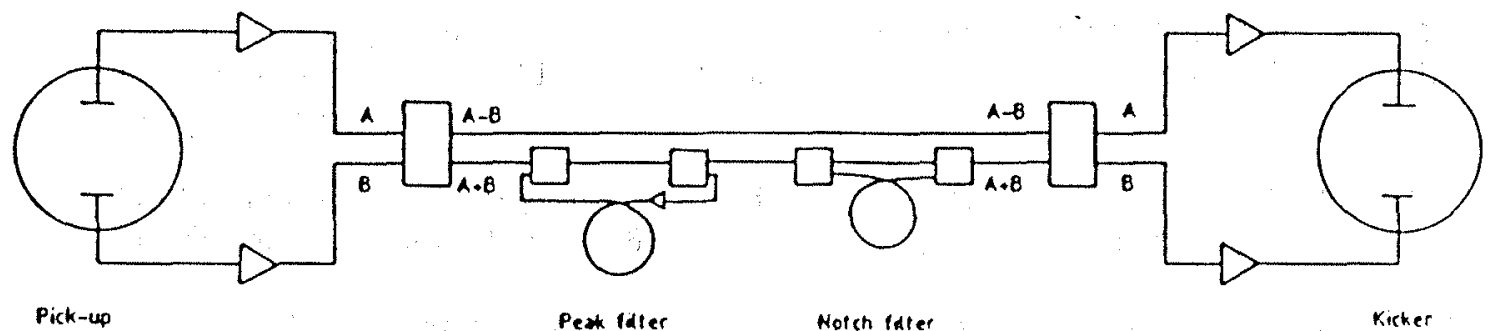

Eiqure 9: Treatment of transverse $(A-B)$ and momentum $(A+B)$ signals between pick-up and kicker. 
assembly. The laboratory measurements are made for the operations in the transverse mode. A complete structure is laid on a ground plate and the particle beam is simulated by a 500 stripline terminated by its characteristic impedance. The frequency response of the structure is obtained using a vector network analyser.

The connection between pick-up and kicker is shown in Fig. 9. After amplification, the pick-up signals are combined in a hybrid circuit so that one gets at the output ports their sum and difference which are proportional to the momentum and position deviations respectively. The converse combination is made at the input to the kicker. Between the two hybrid circuits, the sum signal is processed through two comb filters. The filters are made of two parallel coaxial cables whose difference in electrical length is equal to the circumference of a reference orbit. The transfer functions are therefore periodic with a period equal to the nominal revolution frequency $f_{0}$, they vary like $1-\exp \left(j 2 \pi f / f_{0}\right)$ for the passive notch filter and $\left(1-s \exp \left(j 2 \pi f / f_{0}\right)\right)^{-1}$ for the active peak filter, $s$ being the feedback gain. For the reference frequency $f_{0}$, no signal is transmitted; otherwise a correction is applied to the particles to force their revolution frequency to approach $f_{0}$ so that the particle momentum spread is diminished. This operation could be performed by the notch filter alone but the peak filter which has a broader response accelerates the migration of the particles with a large momentum or frequency deviation towards the reference momentum. It is obviously of prime importance to have a very small frequency dispersion of the notch or peak spacing and of the notch depths. These conditions are fulfilled by equating the ohmic and dielectric losses in the two cables. Last, momentum cooling is not always achieved with the filter method, in the case of the stochastic accumulation, for instance, the particle momentum is detected through the closed orbit position, the signal processing is then the same as for a horizontal transverse cooling.

\section{AMPLIFICATION}

Once the signal has been processed in the most efficient way, a considerable amplification is still needed. In the ACOL and TEVATRON projects, the amplification covers a range of $150 \mathrm{~dB}$ and more for the fast cooling systems; the beam fluctuations and the background noise are indeed of the order of 1 picowatt whereas the power applied to the kicker may exceed 1 kilowatt. All the amplifiers use gallium arsenide field effect transistors and both low noise preamplifiers and power ampliers were the object of thorough developments.

Low noise amplifiers

The low noise preamplifiers have benefited from the pioneering work of radio-astronomers ${ }^{9}$. The special characteristics of the stochastic cooling pre-amplifiers are their broad band and their ultralinear phase response. The first step in the design consists of choosing a bias current which gives an acceptable compromise between a low noise figure and a sufficient gain. Then, the complex input reflection coefficient $r$ is measured, converted into an impedance $Z$ and the input circuit matches the external impedance (508) to the complex conjugate impedance $Z^{*}$ of $Z$. Conductance and susceptance matching are obtained using $1 / 4$ wavelength line and shunt: 
inductors (or open circuited $1 / 8$ wavelength line). In the gigahertz range, lumped elements or coaxial lines may still be preferred to microstrips especially when few amplifiers have to be built. The three-stage Tevatron preamplifier 10 is schematically represented in Fig. 10; its parameters are given in Table 1.

\begin{tabular}{|c|c|c|c|}
\hline$T(0 K)$ & Gain $(d B)$ & Noise Figure $(\mathrm{dB})$ & Phase Error $(\mathrm{d} 0)$ \\
300 & 29 & $(1,1.4)$ & \pm 12 \\
17 & 32 & $(.2, .5)$ & \pm 9 \\
\hline
\end{tabular}

Table 1: Charasterics of TEVATRON low noise preamplifier

Under some simplifying assumptions, the noise temperature $T_{n}$ of a field effect transistor 11 can be expressed by the relation

$$
T_{n}=2 \pi f K T C_{g s} \sqrt{\frac{R_{S}+R_{g}}{g}}
$$

where $f$ is the frequency, $K$ the Fukui factor, $T$ the absolute ambient temperature, $C_{g s}$ the gate-source compacitance, $g$ the transconductance, $R_{5}$ and $R_{g}$ the source and gate resistances. Heterojunction GaAlAs FET's have a Fukui factor as low as 1.55 against 2.5 for usual MESFET's, they are therefore candidates for ultra low noise transistors. As they are built for higher frequency, their characteristics are difficult to determine in our range. Nevertheless, a single stage amplifier using a TEGFET transistor was built by THOMSON CSF and has been tested at CERN; its performances are quite satisfactory in the upper band of the ACOL project $(2.4-3 \mathrm{GHz})$. Gain and phase responses are shown in Fig. 11. The noise temperature becomes difficult to measure when it is very low, it has been estimated to be near $20^{\circ} \mathrm{K}$ for a $30^{\circ} \mathrm{K}$ ambient temperature.

Power amplifiers

Until 1984, broad band microwave power amplifiers were traditionally based upon travelling wave tubes (TWT) especially for continuous operation. However, in our relatively low frequency range, European manufacturers had not an appropriate tube and they were not eager to launch a development program because they estimated that solid state devices would become competitive in a very near future. This prediction was confirmed when, the same year, the Fujitsu field effect transistor FLL 100 MK showed up with an 8 watt output power. It then became conceivable 12 to build 100 watt power amplifiers in the first band of the ACOL project $1-1.6 \mathrm{GHz}$ using a double cascade of paralleled four transistor modules connected by hybrid couplers (Fig. 12). For the second band $(1.6-2.4 \mathrm{GHz})$, a somewhat lower power can be obtained using a four-stage cascade of two transistor modules. The same topology is used for the third band $(2.4-3 \mathrm{GHz}$ ) replacing FLL 100 by FLL 50 transistors which have a higher gain but the output power is about twice as low (Fig. 13).

Based on these concepts, a development program was started at CERN 


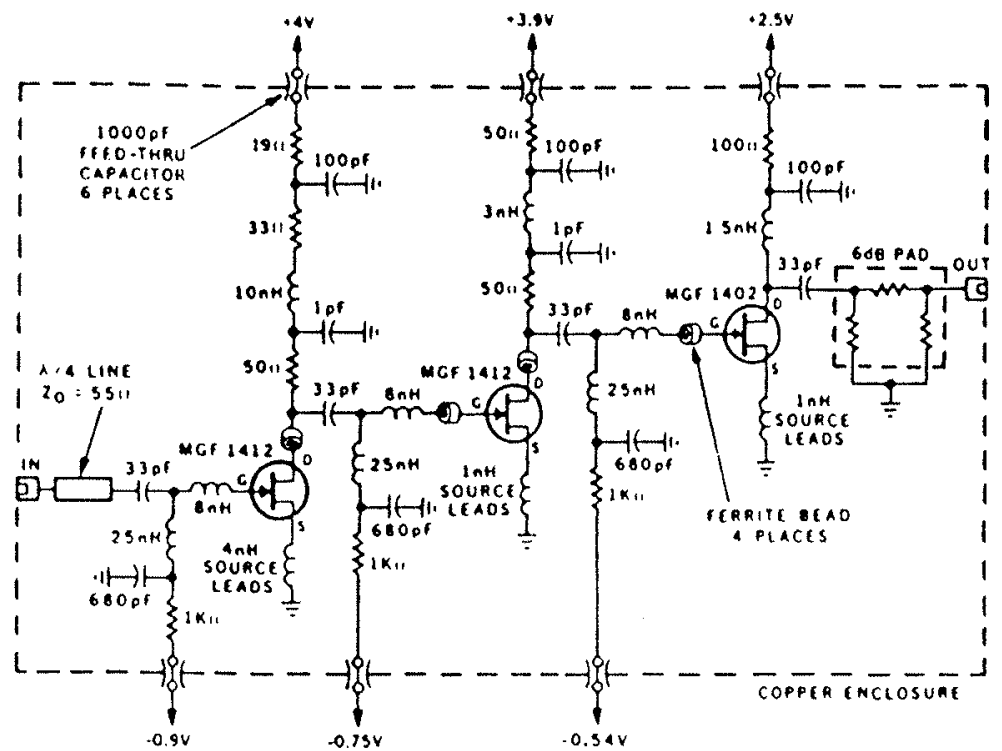

Eiqure 10: Schematic diagram of TEVATRON $1-2 \mathrm{GHz}$ preamplifier.

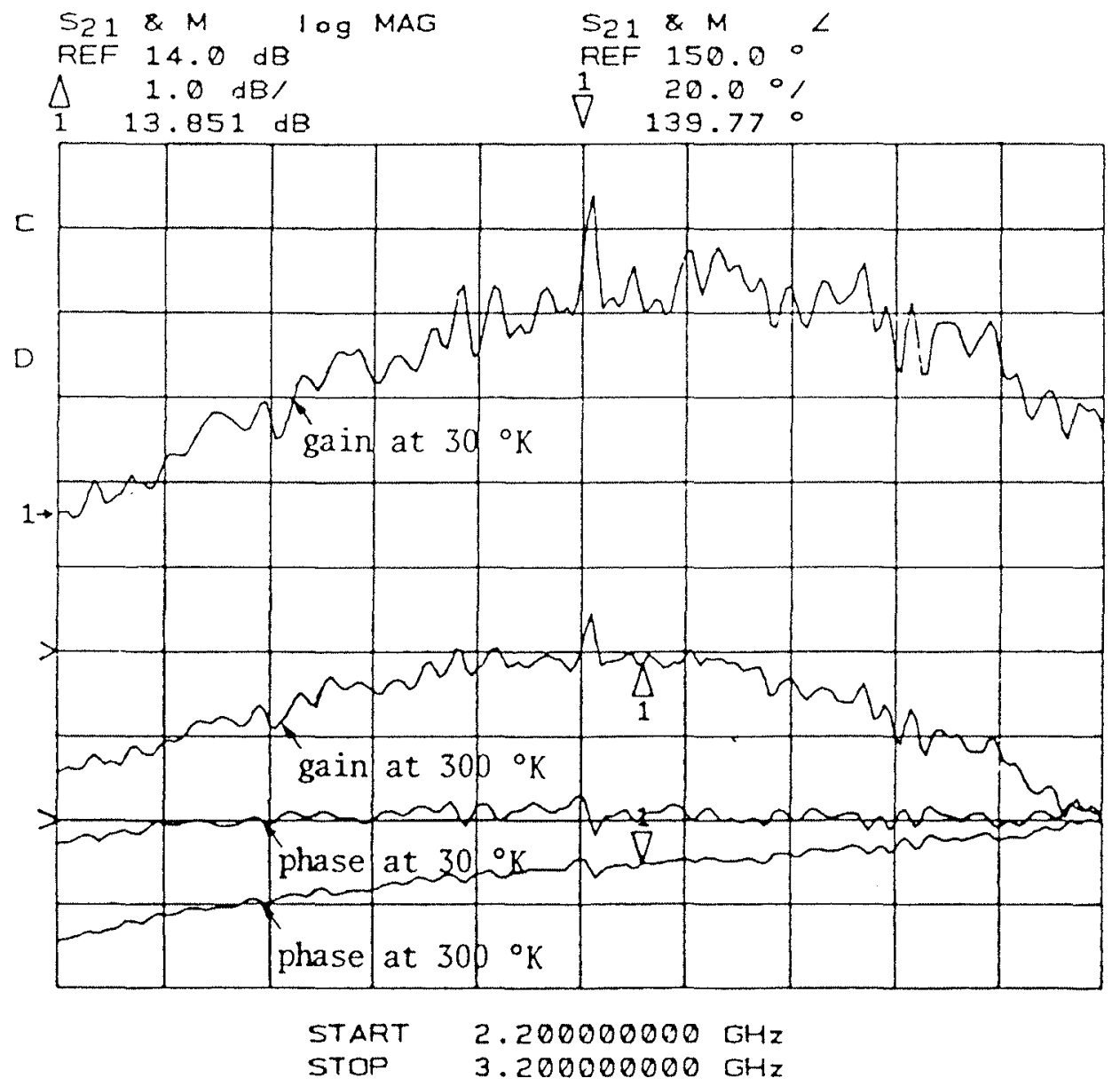

Eiqure 11: Frequency response of an experimental TEGFET preamplifier for ACOL. 


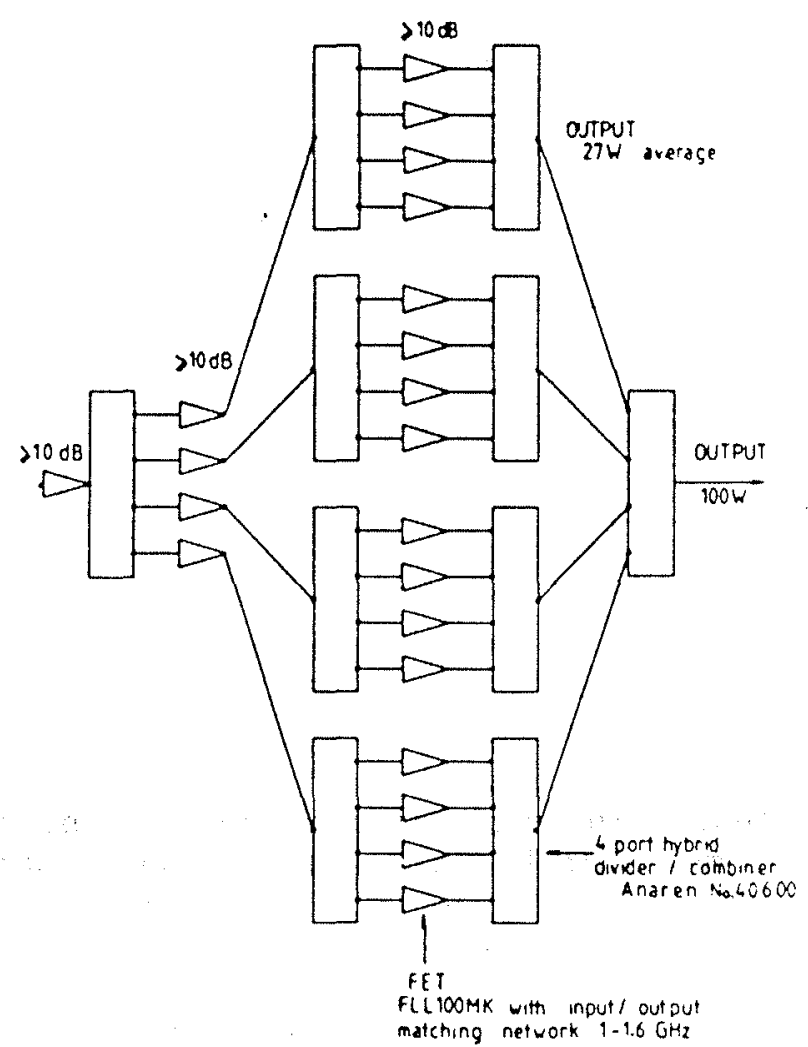

Eigure 12: Schematic layout of a solid state $C W$ power amplifier.

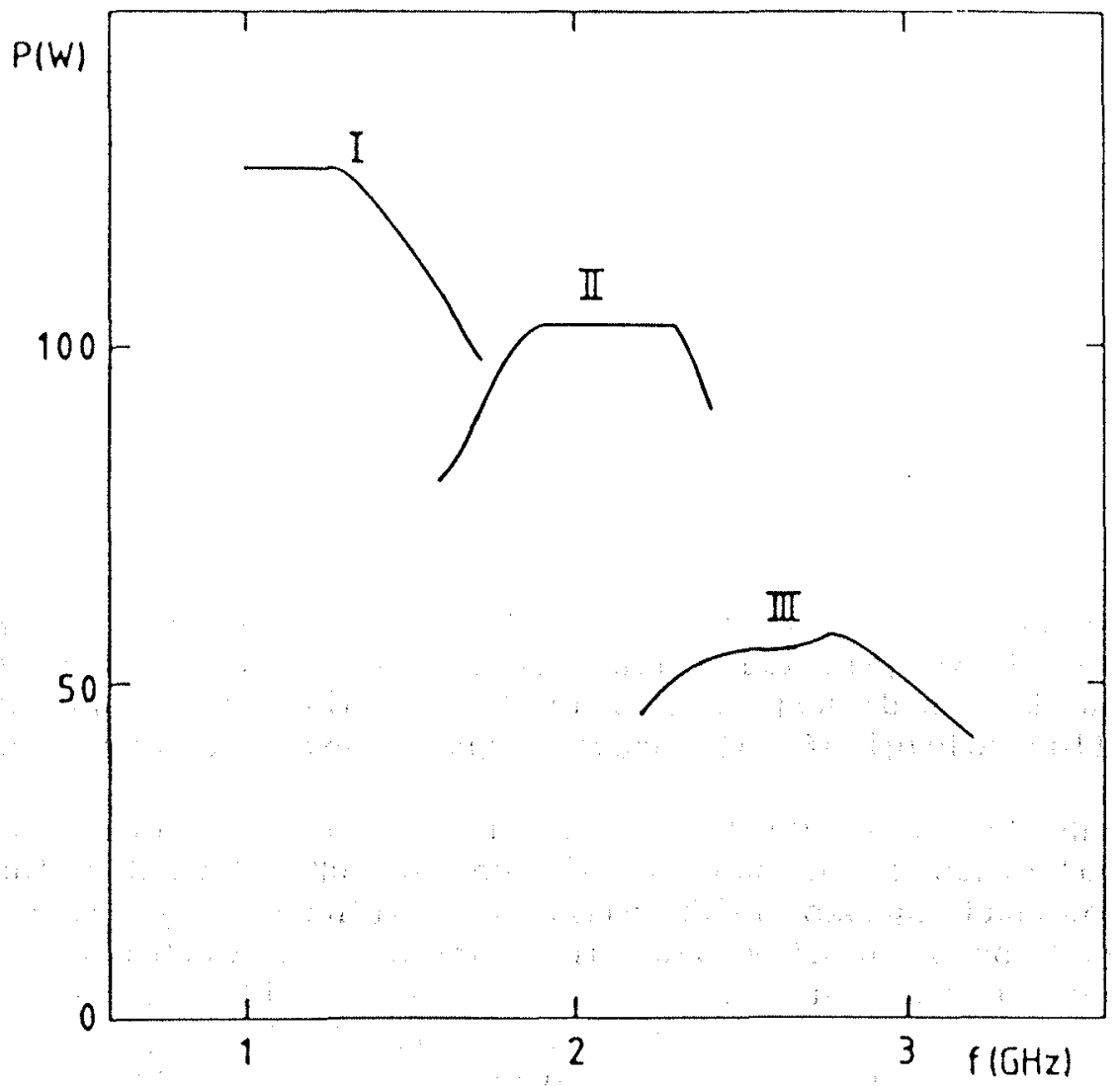

Eiqure 13: Typical output power at $1 \mathrm{~dB}$ compression gain delivered by series power amplifiers. 
and two research contracts were placed in industry for the fabrication of the elementary modules in order to assess the technical specifications and the conditions of a large scale production (130 amplifiers). At the end of 1984, it was decided to adopt the solid state solution and not the TWT's for two reasons. As it has been already emphasized, the phase linearity is of paramount importance in our case; at $1 \mathrm{~dB}$ compression gain, the phase may change by $40^{\circ}$ at least in a TWT with respect to small signal amplitude conditions; with FET's, the phase excursion can be maintained below $10^{\circ}$. In addition, as a result of a comparison with the TEVATRON project which started earlier and was based on TWT amplifiers, it turns out that the cost per watt is cheaper for the FET technology by a factor $2.7,1.7$ and 1.3 for bands I, II and III, respectively.

All the techniques of the low noise amplifiers can be transferred to power amplifiers by taking the output power instead of the noise figure as a factor of merit. Once the best drain voltage is determined, the drain (output) and gate (input) circuits are designed in the large signal conditions; the small signal characteristics are automatically obtained; the converse procedure which consists of optimizing the small signal first is not valid. A delicate item is the output four part combiner of which each way must be carefully balanced to avoid any waste of power. The main parameters of the amplifiers are listed in Table 2.

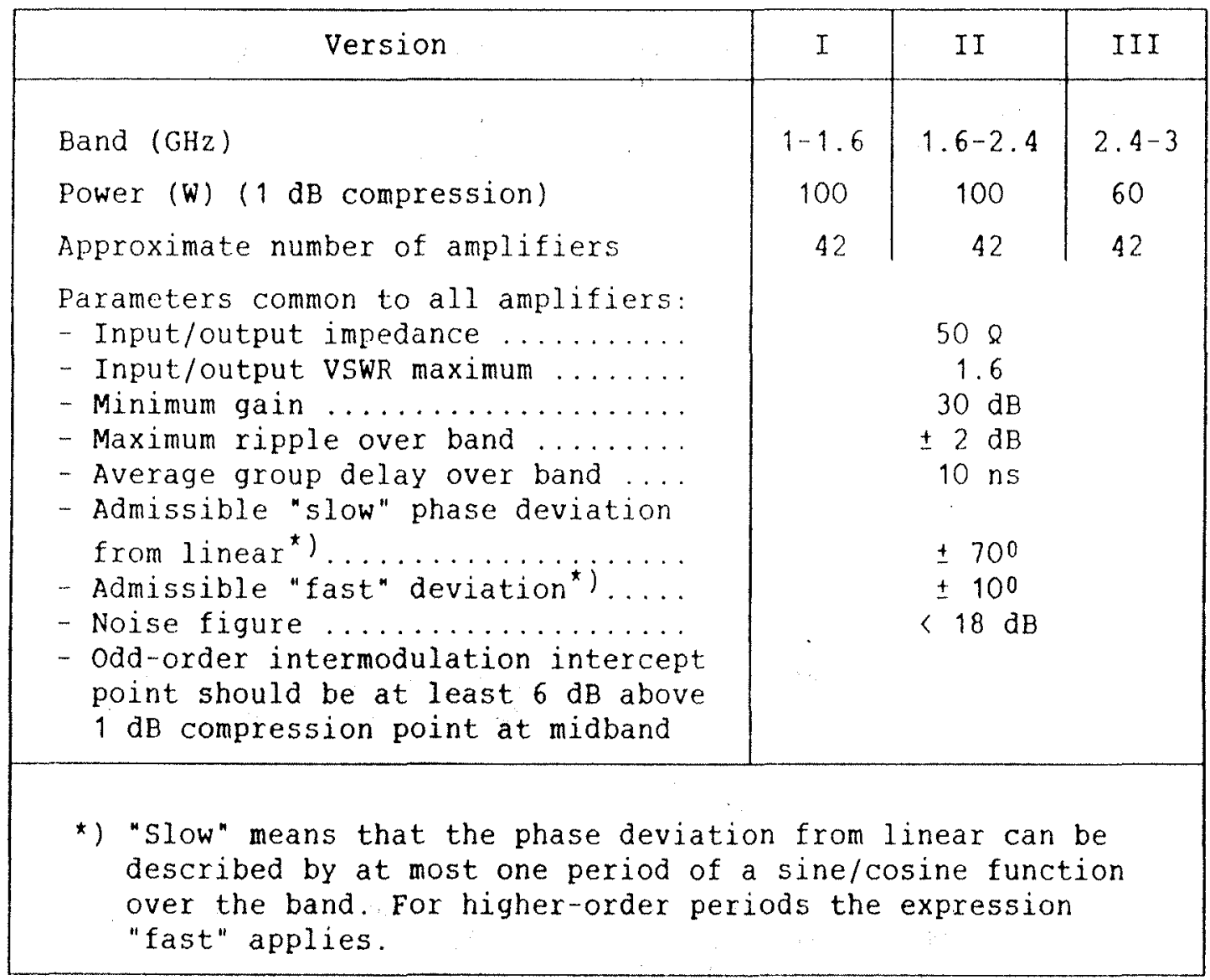

Table 2: Power amplifier parameters 


\section{CONCLUSION}

The invention of stochastic cooling has opened a new realm to microwave techniques in particle accelerators. CERN and the Fermi National ACcelerator Laboratory have given a high priority to antiproton production but a number of other Institutes have applied cooling techniques to other particles, especially to heavy ions. The future trends will be in cooling more intense beams in a shorter time, which means the use of broader bandwidth systems and a shift towards higher frequencies.

Last, the interplay between antimatter physics and technology, especially in the field of solid state devices has been of mutual benefit since, on one hand, systems which were unconceivable ten years ago, have become reality and on the other hand, specific developments, like macrocircuits or solid state power amplifiers should have a broad range of application.

\section{ACKNOWLEDGMENTS}

The modules of the power amplifiers have been developed by DATENO (Dinard-France) and NUCLETUDES (Les Ulis-France). The series power amplifiers have been built by NUCLETUDES.

\section{REFERENCES}

1. van der Meer, S., "Stochastic Cooling and the Accumulation of Antiprotons", Nobel Lecture in Physics, 1984, CERN/PS/84-32 (AA).

2. Möhl, D., Petrucci, G., Thorndahl, L., van der Meer, S. "Physics and Technique of Stochastic Cooling". Physics Reports Vol. 58, Number 2, February 1980 .

3. Design report, Tevatron I project (Fermilab, Batavia, I1l., 1983).

4. Design study of an Antiproton Collector for the Antiproton Accumulator (ACOL), CERN 83-10 (1983).

Autin, B., "The CERN Antiproton Collector", Proceedings of the CERN Accelerator School on Antiprotons for Colliding Beam Facilities, pp. 525-541, October 1983, CERN 84-15.

5. Lefèvre, P., "LEAR", Proceedings of the CERN Accelerator School on Antiprotons for Colliding Beam Facilities, pp. 487-507, October 1983, CERN $84-15$.

6. Lambertson, G. "Dynamic Devices - Pick-ups and Kickers", A.I.P. Conference Proceedings 153, Volume 2, pp. 1413-1442.

7. Autin, B., "Fast Betatron Cooling in an Antiproton. Collector", Proceedings of the Beam Cooling workshop, Madison, September 1982, CERN/PS-AA/82-20.

8. "Micros4 CAD Program", EPFL, Lausanne. 
9. Weinreb, S., Fenstermacher, D., Harris, R. IEEE Trans, on Microwave Theory and Techniques, Volume MTT-30, No. 6, p. 849, June 1982.

10. Leskovar, B., Lo, C.C. IEEE Trans. on Nuclear Science, Volume NS-30, No. 4, August 1983.

11. Delagebeaudeuf, D., Delescluse, P., Jay, P.R. "Extremely Low Noise and LOW Temperature TEGFET Operation", Proceedings of the 15th European Microwave Conference, pp. 260-263, September 1985.

12. Carron, G., Caspers, F., Thorndahl, L. "Development of Power Amplifier Modules for the ACOL Stochastic Cooling Systems", CERN 85-01, February 1985. 